ISSN 1678-3921

Journal homepage: www.embrapa.br/pab

For manuscript submission and journal contents, access: www.scielo.br/pab
Carlos Andre Stuepp ${ }^{(1 凶)}(\mathbb{D}$,

Dagma Kratz(2) (D) ,

Mônica Moreno Gabira(2) (D) and

Ivar Wendling ${ }^{(3)}$ (iD)

(1) Universidade Estadual de Ponta Grossa, Departamento de Fitotecnia e Fitossanidade Campus de Uvaranas, Avenida Carlos Cavalcanti, $n$ ํ 4.748, Uvaranas, CEP 84030-900 Ponta Grossa, PR, Brazil. E-mail: castuepp@uepg.br

(2) Universidade Federal do Paraná, Centro de Ciências Florestais e da Madeira, Campus Jardim Botânico, Avenida Prefeito Lothário Meissner, no 632, Jardim Botânico, CEP 80210-170 Curitiba, PR, Brazil. E-mail: dagmakratz@ufpr.br, monica.gabira@gmail.com

(3) Embrapa Florestas, Estrada da Ribeira, Km 111, Guaraituba, Caixa Postal 319 CEP 83411-000 Colombo, PR, Brazil. E-mail: ivar.wendling@embrapa.br

$\bowtie$ Corresponding author

Received

August 5, 2019

Accepted

June 18, 2020

How to cite

STUEPP, C.A.; KRATZ, D.; GABIRA, M.M.; WENDLING, I. Survival and initial growth in the field of eucalyptus seedlings produced in different substrates. Pesquisa Agropecuária Brasileira, v.55, e01587, 2020. DOI: https://doi. org/10.1590/S1678-3921.pab2020.v55.01587.

\section{Survival and initial growth in the field of eucalyptus seedlings produced in different substrates}

\begin{abstract}
The objective of this work was to evaluate the survival and initial growth, in the field, of eucalyptus seedlings produced in different substrates. Eucalyptus benthamii seedlings produced in 36 substrates were evaluated in the field. The substrates consisted of mixtures, at different volumetric proportions, of: carbonized rice husk, charcoal with granulometry between $1.0-3.0 \mathrm{~mm}$, charcoal with granulometry between $3.0-5.0 \mathrm{~mm}$, coconut fiber, semi-decomposed pine bark, fine vermiculite, sewage sludge, and peat moss. At 1, 2, 3, 6, 12, 18, and 24 months after planting, survival, height, and diameter were determined. At 24 months after planting, height increment, diameter increment, transversal area, and basal area were also measured. The seedlings that showed $100 \%$ survival after 24 months under field conditions were those grown in the nursery in substrates with a higher proportion of fine vermiculite, sewage sludge, and peat moss, that is, in substrates with a higher microporosity. However, there is no effect of substrate characteristics on seedling initial growth in the field. A significant correlation is observed between the survival of seedlings at 6 months and their diameter at the end of the nursery period, evidencing the importance of this characteristic for the establishment of the plant in the field, regardless of the used substrate.
\end{abstract}

Index terms: Eucalyptus benthamii, agricultural residues, forest seedling quality, seedling production, silviculture.

\section{Sobrevivência e crescimento inicial no campo de mudas de eucalipto produzidas em diferentes substratos}

Resumo - O objetivo deste trabalho foi avaliar a sobrevivência e o crescimento inicial, no campo, de mudas de eucalipto produzidas em diferentes substratos. Mudas de Eucalyptus benthamii produzidas em 36 substratos foram avaliadas em campo. Os substratos consistiram em misturas, em diferentes proporções volumétricas, de: casca de arroz carbonizada, carvão com granulometria entre 1,0-3,0 mm, carvão com granulometria entre 3,0-5,0 mm, fibra de coco, casca de pinheiro semidecomposta, vermiculita fina, lodo de esgoto e turfa. Aos 1, 2, 3, 6, 12, 18 e 24 meses após o plantio, foram determinados sobrevivência, altura e diâmetro. Aos 24 meses após o plantio, também foram medidos incremento em altura, incremento em diâmetro, área transversal e área basal. As plântulas que apresentaram sobrevivência de 100\% após 24 meses em condições de campo foram aquelas cultivadas, no viveiro, em substratos com maior proporção de vermiculita fina, lodo de esgoto e turfa, ou seja, em substratos com maior microporosidade. No entanto, não há efeito das características do substrato no crescimento inicial das plântulas. Há correlação significativa entre a sobrevivência da plântula aos 6 meses de idade e seu diâmetro no final da fase de viveiro, o que ressalta a importância desta característica para o estabelecimento da planta em campo, independentemente do substrato utilizado.

Termos para indexação: Eucalyptus benthamii, resíduos agrícolas, qualidade de mudas florestais, produção de mudas, silvicultura. 


\section{Introduction}

Brazil is known worldwide for its excellence in the production of wood biomass, which is the result of favorable environmental conditions (Eufrade Junior et al., 2016) and a management capable of boosting forest research, based on a broad genetic base and breeding programs (Menucelli et al., 2019). The industry that drives the country's bioenergetic production focuses on a set of genetic materials of the genus Eucalyptus (Cavalett et al., 2018). This genus, due to its rapid growth, adaptability, and wood quality, is also widely planted to obtain wood products (Dias Júnior et al., 2016). For this reason, the commercial plantations of Eucalyptus spp. have expanded beyond the traditional producing regions, especially to Southern and Southeastern Brazil. In these two regions, the expansion is attributed to the advance in the selection and consequent recommendation of Eucalyptus species resistant to climatic variations (Elli et al., 2019) and also with a higher productivity than the genus Pinus.

Among the species of Eucalyptus with silviculture potential, Eucalyptus benthamii Maiden \& Cambage stands out as one of the few with frost resistance (Arnold et al., 2015). Its performance has increased the demand for its seedlings, which has led to the emergence of new studies to improve seedling quality, involving the substrate component and its variations (Kratz et al., 2017; Gonzaga et al., 2018; Wang et al., 2018; Gabira et al., 2020). These goals may be achieved by using regional substrate components with low acquisition and transport costs (Stuepp et al., 2016; Mieth et al., 2019; Manca et al., 2020; Yasin et al., 2020). However, it is necessary to pay attention to variations in the physicochemical properties of the formulated substrates, which differ as to their origin, production method, and proportions of used materials (Kratz \& Wendling, 2016; Mieth et al., 2019).

The quality of forest seedlings is closely linked to their survival and initial growth after field planting. Therefore, many studies have focused on determining which factors are most important when producing seedlings with adequate characteristics to be planted in the field (Gonzaga et al., 2018; Wang et al., 2018; Gabira et al., 2020). In general, nursery management including substrates, irrigation, and nutrition - affects directly seedling morphological characteristics, which may result in different responses in the first months of the plantations. Besides seedling size, other factors, such as genetic potential, plant density, climatic conditions, and soil characteristics, may also interfere in plant survival and initial growth after planting (Resquin et al., 2018; Shalizi et al., 2019).

Although the efficiency of alternative substrates has been largely evaluated in nursery conditions (Kratz et al., 2012, 2013; Kratz \& Wendling, 2016; Stuepp et al., 2016; Gabira et al., 2020; Manca et al., 2020), their influence on increasing the growth of forest species in the field has not (Stuepp et al., 2016), generating a gap in substrate recommendation for commercial plantations. The challenge, in this case, has been to produce quality seedlings at a reduced cost (Kratz \& Wendling, 2016; Kratz et al., 2017), seeking a better performance in the field, with reduced mortality and weed competition.

The objective of this work was to evaluate the survival and initial growth, in the field, of Eucalyptus benthamii seedlings produced in different substrates.

\section{Materials and Methods}

Eucalyptus benthamii seedlings were produced in 36 substrates, according to Kratz et al. (2017), for 120 days. The used substrates consisted of mixtures, at different volumetric proportions (v:v), of: carbonized rice husk $(\mathrm{CRH})$, charcoal with granulometry between 1.0-3.0 $\mathrm{mm}(\mathrm{C} 1)$, charcoal with granulometry between 3.0-5.0 $\mathrm{mm}$ (C2), coconut fiber (CF), semi-decomposed pine bark (PB), fine vermiculite (FV), sewage sludge (SS), and peat moss (PM). Two commercial substrates based on peat moss (CS1) and pine bark (CS2) were used as the control. The physical and chemical characteristics of the substrates were determined following the methodology described in Normative Instruction $n^{\circ} 17$ (Brasil, 2007) (Table 1).

The experiment was carried out between June 2014 and June 2016 at Fazenda Canguiri, an experimental area of Universidade Federal do Paraná, located in the municipality of Pinhais, in the state of Paraná, Brazil $\left(25^{\circ} 23^{\prime} \mathrm{S}, 4^{\circ} 07^{\prime} \mathrm{W}\right.$, at $900 \mathrm{~m}$ altitude). According to the classification of Köppen-Geiger (Alvares et al., 2013), the climate of the region is temperate, of the $\mathrm{Cfb}$ type, with temperature between -3 and $22^{\circ} \mathrm{C}$, constant humidity, well-distributed rainfalls throughout the year, and average temperature of the warmest month lower than $22^{\circ} \mathrm{C}$. The soil of the experimental area has a low fertility (Table 2) and was classified as a Cambissolo

Pesq. agropec. bras., Brasília, v.55, e01587, 2020

DOI: 10.1590/S1678-3921.pab2020.v55.01587 
Háplico according to the Brazilian classification system (Santos et al., 2013), i.e., as a Dystric Cambisol in the FAO world reference base for soils (IUSS Working Group WRB, 2015).

Eucalyptus benthamii seeds from a seed production area established in the municipality of Guarapuava, in the state of Paraná, were directly sown in $55-\mathrm{cm}^{3}$ plastic tubes. After sowing, the trays with the tubes were placed, during 120 days, in a greenhouse where irrigation was supplied four times a day for $10 \mathrm{~min}$, at a flow rate of $144 \mathrm{~L} \mathrm{~h}^{-1}$. Fertilization consisted of $4.0 \mathrm{~g} \mathrm{~L}^{-1} \mathrm{CH}_{4} \mathrm{~N}_{2} \mathrm{O}$, $3.0 \mathrm{~g} \mathrm{~L}^{-1}$ simple superphosphate, $0.25 \mathrm{~g} \mathrm{~L}^{-1}$ of the FTE BR 10 fertilizer $(7.0 \% \mathrm{Zn}, 4.0 \% \mathrm{Fe}, 4.0 \% \mathrm{Mn}, 0.1 \% \mathrm{Mo}$, $2.5 \% \mathrm{~B}$, and $0.8 \% \mathrm{Cu}$ ), and $3.0 \mathrm{~g} \mathrm{~L}^{-1} \mathrm{KCl}$, applied every 7 days. After 120 days, seedling height, stem diameter, and shoot and root dry biomass were evaluated, as well as the Dickson quality index (Table 3).

Table 1. Physicochemical characteristics of the substrates used for Eucalyptus benthamii seedling production ${ }^{(1)}$.

\begin{tabular}{|c|c|c|c|c|c|c|}
\hline Substrate (v:v) ${ }^{(2)}$ & $\mathrm{AD}$ & $\mathrm{TP}$ & Microporosity & Macroporosity & $\mathrm{pH}$ & EC \\
\hline & $\left(\mathrm{kg} \mathrm{m}^{-3}\right)$ & -------- & ----(\%)----- & & & $\left(\mathrm{dS} \mathrm{cm} \mathrm{cm}^{-1}\right)$ \\
\hline $10 \mathrm{CRH} / 90 \mathrm{PM}$ & 282.24 & 75.53 & 55.16 & 20.37 & 4.12 & 0.57 \\
\hline $90 \mathrm{SS} / 10 \mathrm{~PB}$ & 547.31 & 71.38 & 52.47 & 18.91 & 9.00 & 4,08 \\
\hline $10 \mathrm{C} 1 / 90 \mathrm{PM}$ & 250.01 & 63.08 & 51.53 & 11.55 & 4.32 & 0.61 \\
\hline $10 \mathrm{CRH} / 90 \mathrm{SS}$ & 542.33 & 74.19 & 50.78 & 23.42 & 9.09 & 4.26 \\
\hline $\mathrm{CS} 1$ & 315.34 & 77.29 & 50.37 & 26.93 & 5.86 & 0.57 \\
\hline $50 \mathrm{SS} / 50 \mathrm{~PB}$ & 470.84 & 71.59 & 48.99 & 22.60 & 8.77 & 3.38 \\
\hline $90 \mathrm{CF} / 10 \mathrm{~PB}$ & 118.85 & 72.74 & 48.32 & 24.43 & 6.51 & 0.13 \\
\hline $50 \mathrm{~PB} / 50 \mathrm{PM}$ & 336.09 & 73.80 & 47.46 & 26.33 & 5.07 & 0.39 \\
\hline $\mathrm{CS} 2$ & 193.09 & 69.06 & 45.06 & 24.00 & 5.26 & 0.93 \\
\hline $50 \mathrm{CF} / 50 \mathrm{~PB}$ & 234.72 & 75.28 & 44.84 & 30.46 & 6.42 & 0.14 \\
\hline $50 \mathrm{C} 1 / 50 \mathrm{SS}$ & 492.70 & 61.74 & 43.67 & 18.07 & 9.22 & 2.88 \\
\hline $50 \mathrm{C} 2 / 50 \mathrm{PM}$ & 312.03 & 58.87 & 43.28 & 15.60 & 5.03 & 0.62 \\
\hline $10 \mathrm{CF} / 90 \mathrm{FV}$ & 150.87 & 68.70 & 43.10 & 25.60 & 7.03 & 0.02 \\
\hline $10 \mathrm{CRH} / 90 \mathrm{FV}$ & 172.30 & 69.57 & 41.80 & 27.19 & 7.39 & 0.02 \\
\hline $50 \mathrm{~PB} / 50 \mathrm{FV}$ & 283.45 & 70.10 & 41.71 & 28.40 & 6.72 & 0.10 \\
\hline $90 \mathrm{~PB} / 10 \mathrm{PM}$ & 355.06 & 78.04 & 41.18 & 36.86 & 6.36 & 0.19 \\
\hline $50 \mathrm{CF} / 50 \mathrm{FV}$ & 120.26 & 67.50 & 40.98 & 26.52 & 6.55 & 0.06 \\
\hline $10 \mathrm{C} 2 / 90 \mathrm{~PB}$ & 353.94 & 75.23 & 40.63 & 34.60 & 6.62 & 0.23 \\
\hline $90 \mathrm{~PB} / 10 \mathrm{FV}$ & 297.15 & 76.88 & 40.24 & 36.64 & 6.49 & 0.19 \\
\hline $10 \mathrm{CRH} / 90 \mathrm{~PB}$ & 339.57 & 79.33 & 39.70 & 39.63 & 6.53 & 0.17 \\
\hline $50 \mathrm{CRH} / 50 \mathrm{PM}$ & 208.29 & 73.61 & 39.41 & 38.34 & 4.49 & 0.32 \\
\hline $50 \mathrm{C} 1 / 50 \mathrm{PM}$ & 197.470 & 63.77 & 38.88 & 24.89 & 5.60 & 0.59 \\
\hline $50 \mathrm{CRH} / 50 \mathrm{SS}$ & 387.65 & 72.16 & 37.41 & 34.75 & 9.12 & 3.62 \\
\hline $50 \mathrm{C} 2 / 50 \mathrm{FV}$ & 228.91 & 55.85 & 35.45 & 20.40 & 8.53 & 0.22 \\
\hline $50 \mathrm{C} 1 / 50 \mathrm{FV}$ & 208.86 & 59.92 & 35.07 & 24.85 & 9.13 & 0.40 \\
\hline $50 \mathrm{C} 2 / 50 \mathrm{~PB}$ & 341.56 & 59.70 & 34.11 & 25.58 & 7.43 & 0.23 \\
\hline $50 \mathrm{CRH} / 50 \mathrm{FV}$ & 160.60 & 69.00 & 33.38 & 33.44 & 7.49 & 0.04 \\
\hline $50 \mathrm{C} 1 / 50 \mathrm{~PB}$ & 311.50 & 66.54 & 32.81 & 33.73 & 7.56 & 0.29 \\
\hline $90 \mathrm{C} 2$ / $10 \mathrm{PM}$ & 298.85 & 49.41 & 31.80 & 17.61 & 7.38 & 0.45 \\
\hline $90 \mathrm{C} 1 / 10 \mathrm{FV}$ & 280.38 & 59.29 & 30.69 & 27.77 & 9.49 & 0.60 \\
\hline $90 \mathrm{C} 2$ / $10 \mathrm{SS}$ & 349.37 & 44.95 & 28.42 & 16.53 & 9.32 & 0.94 \\
\hline $90 \mathrm{C} 1 / 10 \mathrm{~PB}$ & 284.22 & 58.39 & 28.33 & 30.06 & 8.87 & 0.55 \\
\hline $90 \mathrm{C} 1 / 10 \mathrm{SS}$ & 322.84 & 57.29 & 28.16 & 29.13 & 9.18 & 1.99 \\
\hline $50 \mathrm{CRH} / 50 \mathrm{~PB}$ & 204.45 & 73.08 & 26.49 & 46.59 & 6.69 & 0.13 \\
\hline $90 \mathrm{C} 2 / 10 \mathrm{FV}$ & 273.38 & 41.17 & 24.98 & 16.19 & 8.81 & 0.38 \\
\hline $90 \mathrm{CRH} / 10 \mathrm{CF}$ & 86.30 & 69.48 & 16.31 & 53.17 & 7.21 & 0.09 \\
\hline
\end{tabular}

${ }^{(1)} \mathrm{AD}$, apparent density; TP, total porosity; and EC, electric conductivity of the substrates. ${ }^{(2)} \mathrm{CRH}$, carbonized rice husk; PM, peat moss; SS, sewage sludge; PB, semi-decomposed pine bark; $\mathrm{C} 1$, charcoal with granulometry between 1.0-3.0 mm; CS1, commercial substrate based on peat moss; CF, coconut fiber; CS2, commercial substrate based on pine bark; C2, charcoal with granulometry between 3.0-5.0 mm; and FV, fine vermiculite. 
Table 2. Clay content and chemical properties of the Haplic Cambisol of the experimental area in the municipality of Pinhais, in the state of Paraná, Brazil ${ }^{(1)}$.

\begin{tabular}{cccccccccccc}
\hline $\begin{array}{l}\text { Depth } \\
(\mathrm{cm})\end{array}$ & $\begin{array}{c}\mathrm{pH} \\
\mathrm{H}_{2} \mathrm{O}\end{array}$ & $\mathrm{Ca}$ & $\mathrm{Mg}$ & $\begin{array}{c}\mathrm{Al} \\
-------------------\left(\mathrm{cmol}_{\mathrm{c}} \mathrm{dm}^{-3}\right)\end{array}$ & $\mathrm{H}+\mathrm{Al}$ & $\mathrm{CEC}$ & $\mathrm{V}$ & $\mathrm{m}$ & $\begin{array}{c}\mathrm{C} \\
\left(\mathrm{g} \mathrm{dm}^{-3}\right)\end{array}$ & $\begin{array}{c}\mathrm{P} \\
\left(\mathrm{mg} \mathrm{dm}^{-3}\right)\end{array}$ & $\begin{array}{c}\mathrm{K} \\
\left(\mathrm{g} \mathrm{kg}^{-1}\right)\end{array}$ \\
\hline $0-30$ & 5.70 & 8.73 & 3.66 & 0.13 & 4.71 & 17.18 & 73 & 1 & 39.9 & 1.98 & 0.09 \\
$50-80$ & 4.96 & 3.41 & 1.69 & 0.75 & 7.19 & 12.36 & 42 & 13 & 34.0 & 1.20 & 0.07 \\
\hline
\end{tabular}

(1)Used extractors: Mehlich 1 for $\mathrm{P}$ and $\mathrm{K}$; and $\mathrm{KCl}$ for $\mathrm{Ca}, \mathrm{Mg}$, and $\mathrm{Al}$. $\mathrm{CEC}$, cation exchange capacity at $\mathrm{pH}$ 7.0; $\mathrm{V}$, base saturation; and $\mathrm{m}$, aluminum saturation.

Table 3. Stem diameter (D), height (H), H/D ratio, shoot dry biomass (SDB), root dry biomass (RDB), and Dickson quality index (DQI) of Eucalyptus benthamii seedlings at 120 days after sowing in 36 substrates, in greenhouse conditions, in the municipality of Pinhais, in the state of Paraná, Brazil ${ }^{(1)}$.

\begin{tabular}{|c|c|c|c|c|c|c|}
\hline Substrate $^{(2)}$ & Stem diameter $(\mathrm{mm})$ & Height $(\mathrm{cm})$ & $\mathrm{H} / \mathrm{D}$ ratio & SDB (g) & $\mathrm{RDBc}(\mathrm{g})$ & DQI \\
\hline $10 \mathrm{C} 1 / 90 \mathrm{PM}$ & $3.59 \mathrm{a}$ & $34.50 \mathrm{a}$ & $9.61 \mathrm{~b}$ & $1.68 \mathrm{a}$ & $0.68 \mathrm{a}$ & $0.20 \mathrm{a}$ \\
\hline $\mathrm{CS} 1$ & $3.54 \mathrm{a}$ & $31.84 \mathrm{a}$ & $8.99 b$ & $1.53 \mathrm{a}$ & $0.63 \mathrm{a}$ & $0.19 \mathrm{a}$ \\
\hline $10 \mathrm{CRH} / 90 \mathrm{PM}$ & $3.38 \mathrm{a}$ & $34.36 \mathrm{a}$ & $10.16 \mathrm{a}$ & $1.62 \mathrm{a}$ & $0.65 \mathrm{a}$ & $0.18 \mathrm{a}$ \\
\hline $50 \mathrm{~PB} / 50 \mathrm{PM}$ & $3.34 \mathrm{a}$ & $33.55 \mathrm{a}$ & $10.04 \mathrm{~b}$ & $1.57 \mathrm{a}$ & $0.63 \mathrm{a}$ & $0.18 \mathrm{a}$ \\
\hline $50 \mathrm{C} 1 / 50 \mathrm{PM}$ & $3.18 \mathrm{~b}$ & $31.63 \mathrm{a}$ & $9.95 b$ & $1.42 \mathrm{a}$ & $0.58 \mathrm{a}$ & $0.16 \mathrm{~b}$ \\
\hline $50 \mathrm{CRH} / 50 \mathrm{PM}$ & $3.13 b$ & $31.42 \mathrm{a}$ & $10.03 a$ & $1.40 \mathrm{a}$ & $0.57 \mathrm{a}$ & $0.16 \mathrm{~b}$ \\
\hline $50 \mathrm{CRH} / 50 \mathrm{SS}$ & $2.80 \mathrm{~b}$ & $28.22 b$ & $10.08 \mathrm{~b}$ & $1.15 b$ & $0.47 \mathrm{~b}$ & $0.13 \mathrm{~b}$ \\
\hline $10 \mathrm{CRH} / 90 \mathrm{FV}$ & $2.80 \mathrm{~b}$ & $24.68 \mathrm{c}$ & $8.81 \mathrm{a}$ & $0.97 \mathrm{c}$ & $0.41 \mathrm{~b}$ & $0.12 \mathrm{c}$ \\
\hline $10 \mathrm{CF} / 90 \mathrm{FV}$ & $2.61 \mathrm{c}$ & $26.26 \mathrm{~b}$ & $10.07 \mathrm{~b}$ & $0.99 \mathrm{c}$ & $0.41 \mathrm{~b}$ & $0.11 \mathrm{c}$ \\
\hline $\mathrm{CS} 2$ & $2.60 \mathrm{c}$ & $23.82 \mathrm{c}$ & $9.15 b$ & $0.87 \mathrm{c}$ & $0.40 \mathrm{~b}$ & $0.11 \mathrm{c}$ \\
\hline $50 \mathrm{CRH} / 50 \mathrm{~PB}$ & $2.55 \mathrm{c}$ & $26.83 b$ & $10.54 \mathrm{a}$ & $1.01 \mathrm{c}$ & $0.41 \mathrm{~b}$ & $0.11 \mathrm{c}$ \\
\hline $90 \mathrm{CRH} / 10 \mathrm{CF}$ & $2.54 \mathrm{c}$ & $26.05 b$ & $10.27 \mathrm{a}$ & $0.96 \mathrm{c}$ & $0.40 \mathrm{~b}$ & $0.11 \mathrm{c}$ \\
\hline $50 \mathrm{C} 2 / 50 \mathrm{PM}$ & $2.46 \mathrm{c}$ & $27.16 \mathrm{~b}$ & $11.02 \mathrm{~b}$ & $1.00 \mathrm{c}$ & $0.41 \mathrm{~b}$ & $0.11 \mathrm{c}$ \\
\hline $50 \mathrm{SS} / 50 \mathrm{~PB}$ & $2.39 \mathrm{c}$ & $25.80 \mathrm{~b}$ & $10.81 \mathrm{~b}$ & $0.91 \mathrm{c}$ & $0.38 \mathrm{~b}$ & $0.10 \mathrm{c}$ \\
\hline $50 \mathrm{CRH} / 50 \mathrm{FV}$ & $2.38 \mathrm{c}$ & $27.27 b$ & $11.46 \mathrm{a}$ & $0.98 \mathrm{c}$ & $0.40 \mathrm{~b}$ & $0.10 \mathrm{c}$ \\
\hline $10 \mathrm{CRH} / 90 \mathrm{SS}$ & $2.37 \mathrm{c}$ & $29.78 \mathrm{a}$ & $12.56 \mathrm{~b}$ & $1.15 \mathrm{~b}$ & $0.47 \mathrm{~b}$ & $0.11 \mathrm{c}$ \\
\hline $50 \mathrm{C} 2 / 50 \mathrm{FV}$ & $2.32 \mathrm{c}$ & $27.45 b$ & $11.81 \mathrm{a}$ & $0.98 \mathrm{c}$ & $0.40 \mathrm{~b}$ & $0.10 \mathrm{c}$ \\
\hline $50 \mathrm{C} 1 / 50 \mathrm{SS}$ & $2.31 \mathrm{c}$ & $28.39 b$ & $12.31 \mathrm{a}$ & $1.02 \mathrm{c}$ & $0.41 \mathrm{~b}$ & $0.10 \mathrm{c}$ \\
\hline $50 \mathrm{CF} / 50 \mathrm{FV}$ & $2.24 \mathrm{c}$ & $25.74 \mathrm{~b}$ & $11.47 \mathrm{~b}$ & $0.87 \mathrm{c}$ & $0.42 \mathrm{~b}$ & $0.09 \mathrm{c}$ \\
\hline $50 \mathrm{C} 1 / 50 \mathrm{FV}$ & $2.21 \mathrm{c}$ & $26.27 b$ & $11.90 \mathrm{a}$ & $0.88 \mathrm{c}$ & $0.36 \mathrm{~b}$ & $0.09 \mathrm{c}$ \\
\hline $90 \mathrm{C} 2 / 10 \mathrm{SS}$ & $2.19 \mathrm{c}$ & $25.41 \mathrm{~b}$ & $11.60 \mathrm{~b}$ & $0.84 \mathrm{c}$ & $0.34 \mathrm{~b}$ & $0.08 \mathrm{c}$ \\
\hline $50 \mathrm{~PB} / 50 \mathrm{FV}$ & $2.18 \mathrm{c}$ & $22.46 \mathrm{c}$ & $10.32 b$ & $0.68 \mathrm{~d}$ & $0.29 \mathrm{c}$ & $0.08 \mathrm{~d}$ \\
\hline $90 \mathrm{C} 1 / 10 \mathrm{FV}$ & $2.16 \mathrm{c}$ & $21.40 \mathrm{c}$ & $9.91 \mathrm{a}$ & $0.62 \mathrm{~d}$ & $0.27 \mathrm{c}$ & $0.07 \mathrm{~d}$ \\
\hline $90 \mathrm{C} 1 / 10 \mathrm{SS}$ & $2.12 \mathrm{c}$ & $25.83 b$ & $12.19 \mathrm{a}$ & $0.84 \mathrm{c}$ & $0.34 \mathrm{~b}$ & $0.08 \mathrm{c}$ \\
\hline $90 \mathrm{SS} / 10 \mathrm{~PB}$ & $2.11 \mathrm{c}$ & $26.68 b$ & $12.64 \mathrm{~b}$ & $0.90 \mathrm{c}$ & $0.37 \mathrm{~b}$ & $0.09 \mathrm{c}$ \\
\hline $90 \mathrm{~PB} / 10 \mathrm{FV}$ & $2.10 \mathrm{c}$ & $25.26 \mathrm{~b}$ & $12.04 \mathrm{~b}$ & $0.80 \mathrm{c}$ & $0.39 \mathrm{~b}$ & $0.09 \mathrm{c}$ \\
\hline $90 \mathrm{C} 2 / 10 \mathrm{PM}$ & $2.04 d$ & $23.89 \mathrm{c}$ & $11.72 b$ & $0.72 \mathrm{~d}$ & $0.30 \mathrm{c}$ & $0.07 \mathrm{~d}$ \\
\hline $90 \mathrm{C} 1 / 10 \mathrm{~PB}$ & $2.02 \mathrm{~d}$ & $25.40 \mathrm{~b}$ & $12.58 \mathrm{a}$ & $0.79 \mathrm{c}$ & $0.32 \mathrm{~b}$ & $0.07 \mathrm{~d}$ \\
\hline $90 \mathrm{~PB} / 10 \mathrm{PM}$ & $1.92 \mathrm{~d}$ & $20.82 \mathrm{c}$ & $10.83 b$ & $0.53 \mathrm{~d}$ & $0.28 \mathrm{c}$ & $0.06 \mathrm{~d}$ \\
\hline $90 \mathrm{CF} / 10 \mathrm{~PB}$ & $1.84 \mathrm{~d}$ & $17.93 \mathrm{c}$ & $9.72 b$ & $0.36 \mathrm{~d}$ & $0.18 \mathrm{c}$ & $0.05 \mathrm{~d}$ \\
\hline $50 \mathrm{CF} / 50 \mathrm{~PB}$ & $1.83 \mathrm{~d}$ & $19.64 \mathrm{c}$ & $10.74 \mathrm{~b}$ & $0.99 \mathrm{c}$ & $0.36 \mathrm{~b}$ & $0.10 \mathrm{c}$ \\
\hline $10 \mathrm{C} 2 / 90 \mathrm{~PB}$ & $1.80 \mathrm{~d}$ & $22.83 \mathrm{c}$ & $12.70 \mathrm{a}$ & $0.60 \mathrm{~d}$ & $0.30 \mathrm{c}$ & $0.06 \mathrm{~d}$ \\
\hline $10 \mathrm{CRH} / 90 \mathrm{~PB}$ & $1.79 \mathrm{~d}$ & $18.29 \mathrm{c}$ & $10.23 \mathrm{a}$ & $0.36 \mathrm{~d}$ & $0.20 \mathrm{c}$ & $0.05 \mathrm{~d}$ \\
\hline $90 \mathrm{C} 2 / 10 \mathrm{FV}$ & $1.77 \mathrm{~d}$ & $21.34 \mathrm{c}$ & $12.09 \mathrm{a}$ & $0.51 \mathrm{~d}$ & $0.22 \mathrm{c}$ & $0.05 \mathrm{~d}$ \\
\hline $50 \mathrm{C} 1 / 50 \mathrm{~PB}$ & $1.71 \mathrm{~d}$ & $21.75 \mathrm{c}$ & $12.70 \mathrm{a}$ & $0.52 \mathrm{~d}$ & $0.22 \mathrm{c}$ & $0.05 \mathrm{~d}$ \\
\hline $50 \mathrm{C} 2 / 50 \mathrm{~PB}$ & $1.65 \mathrm{~d}$ & $20.08 \mathrm{c}$ & $12.14 \mathrm{a}$ & $0.42 d$ & $0.19 \mathrm{c}$ & $0.04 \mathrm{~d}$ \\
\hline
\end{tabular}

${ }^{(1)}$ Means followed by equal letters, in the columns, do not differ by Scott-Knott's test, at $5 \%$ probability. ${ }^{(2)} \mathrm{C} 1$, charcoal with granulometry between 1.0-3.0 mm; PM, peat moss; CS1, commercial substrate based on peat moss; CRH, carbonized rice husk; PB, semi-decomposed pine bark; SS, sewage sludge; FV, fine vermiculite; CF, coconut fiber; CS2, commercial substrate based on pine bark; and C2, charcoal with granulometry between 3.0-5.0 mm. 
Before seedling plantation in the field, mechanical mowing was conducted in the total area of the experiment, followed by the control of leaf-cutting ants with a natural bait made with orange peel (Perri et al., 2017). The planting lines were marked with a subsoiler at a $50-\mathrm{cm}$ depth, with a spacing of $3.0 \mathrm{~m}$ between the lines and $1.5 \mathrm{~m}$ in the line. A manual planter was used for sowing in July 2014, when $100 \mathrm{~g}$ of the $\mathrm{N}_{-} \mathrm{P}_{2} \mathrm{O}_{5^{-}}$ $\mathrm{K}_{2} \mathrm{O}$ (4-14-8) fertilizer were applied per pit.

Post-planting cultural practices consisted of mechanical mowing every 6 months and the control of leaf-cutting ants whenever necessary. Seedling survival was evaluated at $1,2,3,6,12,18$, and 24 months after planting, while base diameter at $10 \mathrm{~cm}$ from the soil and total plant height were assessed at 6 , 12,18 , and 24 months after planting in the field.

At the end of the plant measurement period, height increment and diameter increment were also determined. For this, the values measured at 24 months after planting were subtracted from those obtained at planting.

From the base diameter at 24 months, the transversal area and the basal area were estimated, considering 2,500 plants per hectare.

The experiment was set in a randomized complete block design, with 36 treatments and five blocks of three plants per plot. Data were checked for normality by Shapiro-Wilk's test, at 5\% probability, and for homogeneity of variances by Bartlett's test, also at $5 \%$ probability, and, then, subjected to the analysis of variance. Averages were compared by Scott-Knott's test, at 5\% probability. For survival and field growth evaluations, a split-plot design was used. Pearson's correlation analysis was applied to compare the effects of the morphological characteristics of seedlings, substrate characteristics, and plant survival and growth in the field.

\section{Results and Discussion}

Seedling survival differed significantly between treatments at $6,12,18$, and 24 months after planting in the field (Table 4). The lowest survival at 24 months was $60 \%$ for seedlings produced in the $90 \mathrm{C} 1 / 10 \mathrm{~PB}$, 50C $2 / 50 \mathrm{~PB}, 10 \mathrm{C} 2 / 90 \mathrm{~PB}$, and 50C2/50FV substrates. At the same evaluation time, $100 \%$ survival was observed for seedlings grown in 10CRH/90FV, 90SS/10PB, 10C1/90PM, and 50C2/50PM. Therefore, the survival rate was higher for seedlings produced in substrates with a higher microporosity, probably due to the plant's better growth in diameter and the substrate's higher water holding capacity in the first days after planting. Shalizi et al. (2019) also reported the relationship between the initial growth of $E$. benthamii and substrates with a higher microporosity, when subjecting the seedlings produced in substrates with different physical characteristics to drought stress.

Similar growth tendencies were found for height and diameter (Figures 1 and 2). At 18 and 24 months, both variables increased with seedling growth. Different growth tendencies were only observed for seedlings produced in substrates $90 \mathrm{C} 1 / 10 \mathrm{SS}, 50 \mathrm{C} 2 / 50 \mathrm{FV}$, and 10CRH/90FV. Seedling morphological characteristics at the time of field planting directly affected their diameter at 24 months; however, substrate characteristics did not affect the initial growth of the seedlings. Seedling size is important for predicting the success of a plantation because it is directly related to the plant's capacity to overcome weed competition and develop new roots (Khanal et al., 2018; Riikonen \& Luoranen, 2018). It should be noted that, compared with diameter, height seems to be much more dependent on environmental factors, such as weed competition and plant density (Resquin et al., 2018).

As a reflect of growth on diameter, transversal and basal area followed the same trend at 24 months after field planting (Table 5). Knowing the performance of forest species seedlings in the field is an essential tool to determine the best cultural practices to be adopted in nurseries for a better morphophysiological performance after planting. According to Grossnickle \& El-Kassaby (2016), most morphological attributes are nondestructive, easy to measure, and reliable to establish qualitative standards for seedlings. In fact, in the present study, morphological characteristics, such as stem diameter, height, and shoot and root dry biomass, had a positive effect on the increase in individual and total basal area.

The results of Pearson's correlation confirm the importance of seedling diameter at the end of the nursery production phase (Table 6). The significant correlation between the survival of seedlings at 6 months and their diameter at the end of the nursery period evidences the importance of this characteristic for the establishment of the plant in the field. However, after field planting, height and diameter were only 
Table 4. Survival of Eucalyptus benthamii seedlings produced in 36 substrates at 1, 2, 3, 6, 12, 18, and 24 months after planting in the field, in the municipality of Pinhais, in the state of Paraná, Brazil ${ }^{(1)}$.

\begin{tabular}{|c|c|c|c|c|c|c|c|}
\hline \multirow[t]{2}{*}{ Substrate $^{(2)}$} & \multicolumn{7}{|c|}{ Survival (\%) } \\
\hline & 1 month & 2 months & 3 months & 6 months & 12 months & 18 months & 24 months \\
\hline $10 \mathrm{CRH} / 90 \mathrm{FV}$ & $100.00 \mathrm{aA}$ & $100.00 \mathrm{aA}$ & $100.00 \mathrm{aA}$ & $100.00 \mathrm{aA}$ & $100.00 \mathrm{aA}$ & $100.00 \mathrm{aA}$ & $100.00 \mathrm{aA}$ \\
\hline $90 \mathrm{SS} / 10 \mathrm{~PB}$ & $100.00 \mathrm{aA}$ & $100.00 \mathrm{aA}$ & $100.00 \mathrm{aA}$ & $100.00 \mathrm{aA}$ & $100.00 \mathrm{aA}$ & $100.00 \mathrm{aA}$ & $100.00 \mathrm{aA}$ \\
\hline $10 \mathrm{C} 1 / 90 \mathrm{PM}$ & $100.00 \mathrm{aA}$ & $100.00 \mathrm{aA}$ & $100.00 \mathrm{aA}$ & $100.00 \mathrm{aA}$ & $100.00 \mathrm{aA}$ & $100.00 \mathrm{aA}$ & $100.00 \mathrm{aA}$ \\
\hline $50 \mathrm{C} 2 / 50 \mathrm{PM}$ & $100.00 \mathrm{aA}$ & $100.00 \mathrm{aA}$ & $100.00 \mathrm{aA}$ & $100.00 \mathrm{aA}$ & $100.00 \mathrm{aA}$ & $100.00 \mathrm{aA}$ & $100.00 \mathrm{aA}$ \\
\hline $10 \mathrm{CRH} / 90 \mathrm{PM}$ & $100.00 \mathrm{aA}$ & $100.00 \mathrm{aA}$ & $100.00 \mathrm{aA}$ & $93.33 \mathrm{aA}$ & $93.33 \mathrm{aA}$ & $93.33 \mathrm{aA}$ & $93.33 \mathrm{aA}$ \\
\hline $90 \mathrm{C} 2$ / $10 \mathrm{SS}$ & $100.00 \mathrm{aA}$ & $100.00 \mathrm{aA}$ & $100.00 \mathrm{aA}$ & $93.33 \mathrm{aA}$ & $93.33 \mathrm{aA}$ & $93.33 \mathrm{aA}$ & $93.33 \mathrm{aA}$ \\
\hline $50 \mathrm{~PB} / 50 \mathrm{PM}$ & $100.00 \mathrm{aA}$ & $100.00 \mathrm{aA}$ & $100.00 \mathrm{aA}$ & $93.33 \mathrm{aA}$ & $93.33 \mathrm{aA}$ & $93.33 \mathrm{aA}$ & $93.33 \mathrm{aA}$ \\
\hline CS2 & $100.00 \mathrm{aA}$ & $100.00 \mathrm{aA}$ & $100.00 \mathrm{aA}$ & $93.33 \mathrm{aA}$ & $93.33 \mathrm{aA}$ & $93.33 \mathrm{aA}$ & $93.33 \mathrm{aA}$ \\
\hline $90 \mathrm{~PB} / 10 \mathrm{FV}$ & $100.00 \mathrm{aA}$ & $100.00 \mathrm{aA}$ & $100.00 \mathrm{aA}$ & $93.33 \mathrm{aA}$ & $93.33 \mathrm{aA}$ & $86.67 \mathrm{aA}$ & $86.67 \mathrm{aA}$ \\
\hline CS1 & $100.00 \mathrm{aA}$ & $100.00 \mathrm{aA}$ & $100.00 \mathrm{aA}$ & $93.33 \mathrm{aA}$ & $93.33 \mathrm{aA}$ & $86.67 \mathrm{aA}$ & $86.67 \mathrm{aA}$ \\
\hline $10 \mathrm{CRH} / 90 \mathrm{SS}$ & $100.00 \mathrm{aA}$ & $100.00 \mathrm{aA}$ & $100.00 \mathrm{aA}$ & $93.33 \mathrm{aA}$ & $86.67 \mathrm{aA}$ & $86.67 \mathrm{aA}$ & $86.67 \mathrm{aA}$ \\
\hline $50 \mathrm{CRH} / 50 \mathrm{PM}$ & $100.00 \mathrm{aA}$ & $100.00 \mathrm{aA}$ & $100.00 \mathrm{aA}$ & $86.67 \mathrm{aA}$ & $86.67 \mathrm{aA}$ & $86.67 \mathrm{aA}$ & $86.67 \mathrm{aA}$ \\
\hline $50 \mathrm{C} 1 / 50 \mathrm{PM}$ & $100.00 \mathrm{aA}$ & $93.33 \mathrm{aA}$ & $93.33 \mathrm{aA}$ & $86.67 \mathrm{aA}$ & $86.67 \mathrm{aA}$ & $86.67 \mathrm{aA}$ & $86.67 \mathrm{aA}$ \\
\hline $50 \mathrm{C} 1 / 50 \mathrm{~PB}$ & $100.00 \mathrm{aA}$ & $100.00 \mathrm{aA}$ & $100.00 \mathrm{aA}$ & $86.67 \mathrm{aA}$ & $86.67 \mathrm{aA}$ & $86.67 \mathrm{aA}$ & $80.00 \mathrm{aA}$ \\
\hline $10 \mathrm{CRH} / 90 \mathrm{~PB}$ & $100.00 \mathrm{aA}$ & $100.00 \mathrm{aA}$ & $100.00 \mathrm{aA}$ & $80.00 \mathrm{bB}$ & $80.00 \mathrm{bB}$ & $80.00 \mathrm{bA}$ & $80.00 \mathrm{bA}$ \\
\hline $50 \mathrm{CF} / 50 \mathrm{FV}$ & $100.00 \mathrm{aA}$ & $100.00 \mathrm{aA}$ & $100.00 \mathrm{aA}$ & $80.00 \mathrm{bB}$ & $80.00 \mathrm{bB}$ & $80.00 \mathrm{bA}$ & $80.00 \mathrm{bA}$ \\
\hline $90 \mathrm{CRH} / 10 \mathrm{CF}$ & $100.00 \mathrm{aA}$ & $86.67 \mathrm{aA}$ & $86.67 \mathrm{aA}$ & $80.00 \mathrm{aB}$ & $80.00 \mathrm{aB}$ & $80.00 \mathrm{aA}$ & $80.00 \mathrm{aA}$ \\
\hline $10 \mathrm{CF} / 90 \mathrm{FV}$ & $100.00 \mathrm{aA}$ & $100.00 \mathrm{aA}$ & $100.00 \mathrm{aA}$ & $86.67 \mathrm{bA}$ & $80.00 \mathrm{bB}$ & $73.33 \mathrm{bB}$ & $73.33 \mathrm{bB}$ \\
\hline $90 \mathrm{C} 1 / 10 \mathrm{SS}$ & $100.00 \mathrm{aA}$ & $100.00 \mathrm{aA}$ & $100.00 \mathrm{aA}$ & $86.67 \mathrm{bA}$ & $73.33 \mathrm{bB}$ & $73.33 \mathrm{bB}$ & $73.33 \mathrm{bB}$ \\
\hline $50 \mathrm{CRH} / 50 \mathrm{FV}$ & $100.00 \mathrm{aA}$ & $100.00 \mathrm{aA}$ & $100.00 \mathrm{aA}$ & $80.00 \mathrm{bB}$ & $73.33 \mathrm{bB}$ & $73.33 \mathrm{bB}$ & $73.33 \mathrm{bB}$ \\
\hline $50 \mathrm{SS} / 50 \mathrm{~PB}$ & $100.00 \mathrm{aA}$ & $100.00 \mathrm{aA}$ & $100.00 \mathrm{aA}$ & $80.00 \mathrm{bB}$ & $73.33 \mathrm{bB}$ & $73.33 \mathrm{bB}$ & $73.33 \mathrm{bB}$ \\
\hline $90 \mathrm{~PB} / 10 \mathrm{PM}$ & $100.00 \mathrm{aA}$ & $100.00 \mathrm{aA}$ & $100.00 \mathrm{aA}$ & $73.33 \mathrm{bB}$ & $73.33 \mathrm{bB}$ & $73.33 \mathrm{bB}$ & $73.33 \mathrm{bB}$ \\
\hline $50 \mathrm{C} 1 / 50 \mathrm{FV}$ & $100.00 \mathrm{aA}$ & $100.00 \mathrm{aA}$ & $93.33 \mathrm{aA}$ & $80.00 \mathrm{bB}$ & $80.00 \mathrm{bB}$ & $73.33 \mathrm{bB}$ & $73.33 \mathrm{bB}$ \\
\hline $50 \mathrm{C} 1 / 50 \mathrm{SS}$ & $100.00 \mathrm{aA}$ & $93.33 \mathrm{aA}$ & $93.33 \mathrm{aA}$ & $80.00 \mathrm{aB}$ & $80.00 \mathrm{aB}$ & $80.00 \mathrm{aA}$ & $73.33 \mathrm{aB}$ \\
\hline $50 \mathrm{CRH} / 50 \mathrm{~PB}$ & $100.00 \mathrm{aA}$ & $93.33 \mathrm{aA}$ & $93.33 \mathrm{aA}$ & $73.33 \mathrm{bB}$ & $73.33 \mathrm{bB}$ & $73.33 \mathrm{bB}$ & $73.33 \mathrm{bB}$ \\
\hline $90 \mathrm{CF} / 10 \mathrm{~PB}$ & $100.00 \mathrm{aA}$ & $86.67 \mathrm{aA}$ & $86.67 \mathrm{aA}$ & $80.00 \mathrm{aB}$ & $73.33 \mathrm{aB}$ & $73.33 \mathrm{aB}$ & $73.33 \mathrm{aB}$ \\
\hline $50 \mathrm{~PB} / 50 \mathrm{FV}$ & $100.00 \mathrm{aA}$ & $80.00 \mathrm{aA}$ & $80.00 \mathrm{aA}$ & $73.33 \mathrm{aB}$ & $73.33 \mathrm{aB}$ & $73.33 \mathrm{aB}$ & $73.33 \mathrm{aB}$ \\
\hline $90 \mathrm{C} 2 / 10 \mathrm{FV}$ & $100.00 \mathrm{aA}$ & $100.00 \mathrm{aA}$ & $100.00 \mathrm{aA}$ & $80.00 \mathrm{bB}$ & $73.33 \mathrm{bB}$ & $66.67 \mathrm{bB}$ & $66.67 \mathrm{bB}$ \\
\hline $90 \mathrm{C} 1 / 10 \mathrm{FV}$ & $100.00 \mathrm{aA}$ & $93.33 \mathrm{aA}$ & $93.33 \mathrm{aA}$ & $80.00 \mathrm{bB}$ & $66.67 \mathrm{bB}$ & $66.67 \mathrm{bB}$ & $66.67 \mathrm{bB}$ \\
\hline $50 \mathrm{CRH} / 50 \mathrm{SS}$ & $100.00 \mathrm{aA}$ & $93.33 \mathrm{aA}$ & $93.33 \mathrm{aA}$ & $73.33 \mathrm{bB}$ & $73.33 \mathrm{bB}$ & $66.67 \mathrm{bB}$ & $66.67 \mathrm{bB}$ \\
\hline $50 \mathrm{CF} / 50 \mathrm{~PB}$ & $100.00 \mathrm{aA}$ & $86.67 \mathrm{aA}$ & $86.67 \mathrm{aA}$ & $66.67 \mathrm{bB}$ & $66.67 \mathrm{bB}$ & $66.67 \mathrm{bB}$ & $66.67 \mathrm{bB}$ \\
\hline $50 \mathrm{C} 2 / 50 \mathrm{FV}$ & $100.00 \mathrm{aA}$ & $100.00 \mathrm{aA}$ & $100.00 \mathrm{aA}$ & $80.00 \mathrm{bB}$ & $73.33 \mathrm{bB}$ & $73.33 \mathrm{bB}$ & $60.00 \mathrm{bB}$ \\
\hline $10 \mathrm{C} 2$ / $90 \mathrm{~PB}$ & $100.00 \mathrm{aA}$ & $100.00 \mathrm{aA}$ & $100.00 \mathrm{aA}$ & $73.33 \mathrm{bB}$ & $66.67 \mathrm{bB}$ & $60.00 \mathrm{bB}$ & $60.00 \mathrm{bB}$ \\
\hline $90 \mathrm{C} 1 / 10 \mathrm{~PB}$ & $100.00 \mathrm{aA}$ & $86.67 \mathrm{aA}$ & $86.67 \mathrm{aA}$ & $66.67 \mathrm{bB}$ & $60.00 \mathrm{bB}$ & $60.00 \mathrm{bB}$ & $60.00 \mathrm{bB}$ \\
\hline $50 \mathrm{C} 2 / 50 \mathrm{~PB}$ & $100.00 \mathrm{aA}$ & $86.67 \mathrm{aA}$ & $73.33 \mathrm{bA}$ & $60.00 \mathrm{bB}$ & $60.00 \mathrm{bB}$ & $60.00 \mathrm{bB}$ & $60.00 \mathrm{bB}$ \\
\hline $90 \mathrm{C} 2 / 10 \mathrm{PM}$ & $100.00 \mathrm{aA}$ & $93.33 \mathrm{aA}$ & $86.67 \mathrm{aA}$ & $58.33 \mathrm{bB}$ & $58.33 \mathrm{bB}$ & $58.33 \mathrm{bB}$ & $58.33 \mathrm{bB}$ \\
\hline
\end{tabular}

${ }^{(1)}$ Means followed by equal letters, lowercase in the rows and uppercase in the columns, do not differ from each other by Scott-Knott's test, at $5 \%$ probability. ${ }^{(2)} \mathrm{CRH}$, carbonized rice husk; FV, fine vermiculite SS, sewage sludge; PB, semi-decomposed pine bark; C1, charcoal with granulometry between 1.0-3.0 mm; PM, peat moss; C2, charcoal with granulometry between 3.0-5.0 mm; CS2, commercial substrate based on pine bark; CS1, commercial substrate based on peat moss; and $\mathrm{CF}$, coconut fiber. 

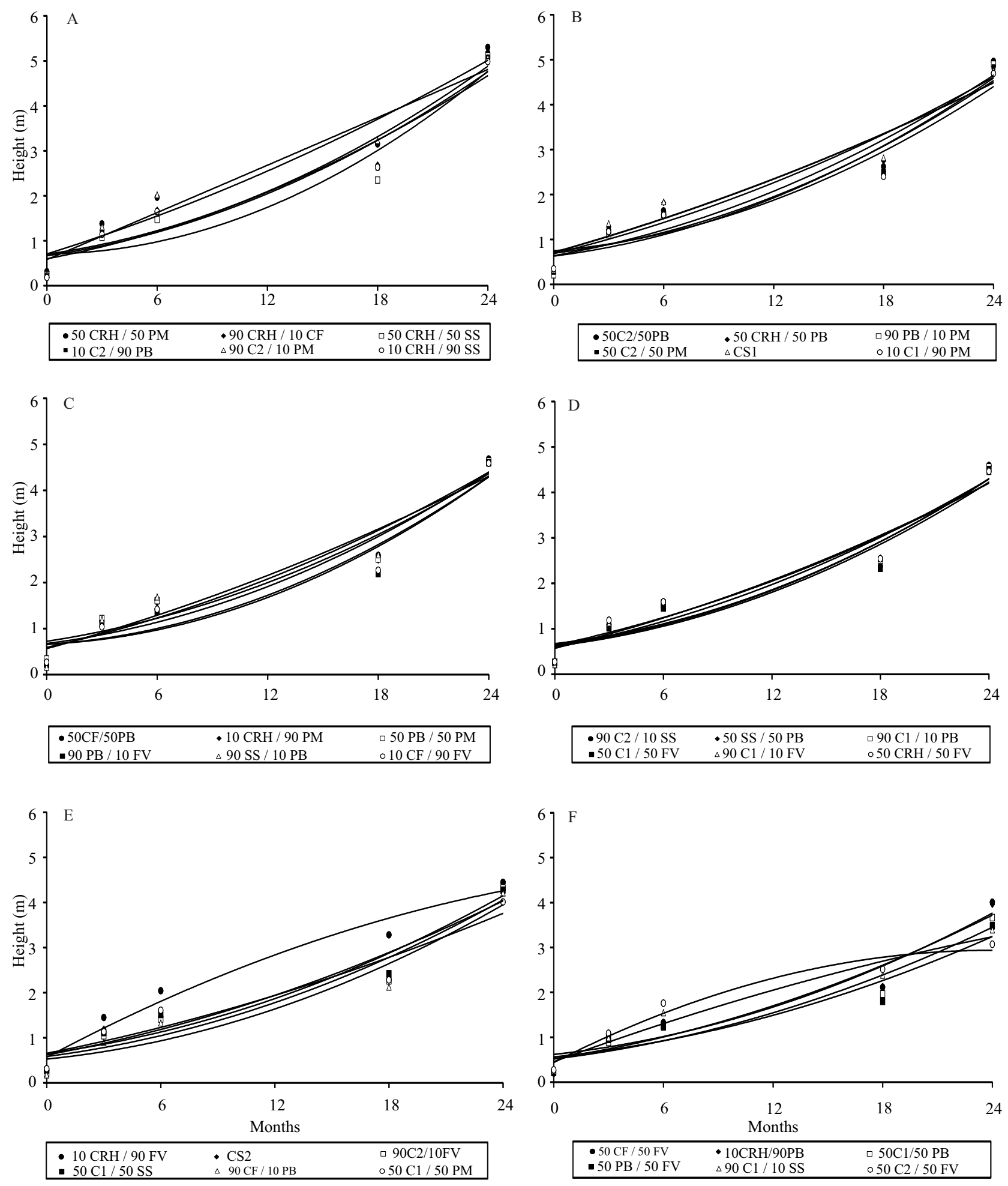

Figure 1. Growth trend for the height of Eucalyptus benthamii seedlings produced in 36 substrates and average height at 24 months after planting in the field. $\mathrm{CRH}$, carbonized rice husk; PM, peat moss; CF, coconut fiber; SS, sewage sludge; $\mathrm{C} 2$, charcoal with granulometry between $3.0-5.0 \mathrm{~mm}$; PB, semi-decomposed pine bark; CS1, commercial substrate based on peat moss; C1, charcoal with granulometry between 1.0-3.0 mm; FV, fine vermiculite; and CS2, commercial substrate based on pine bark. 

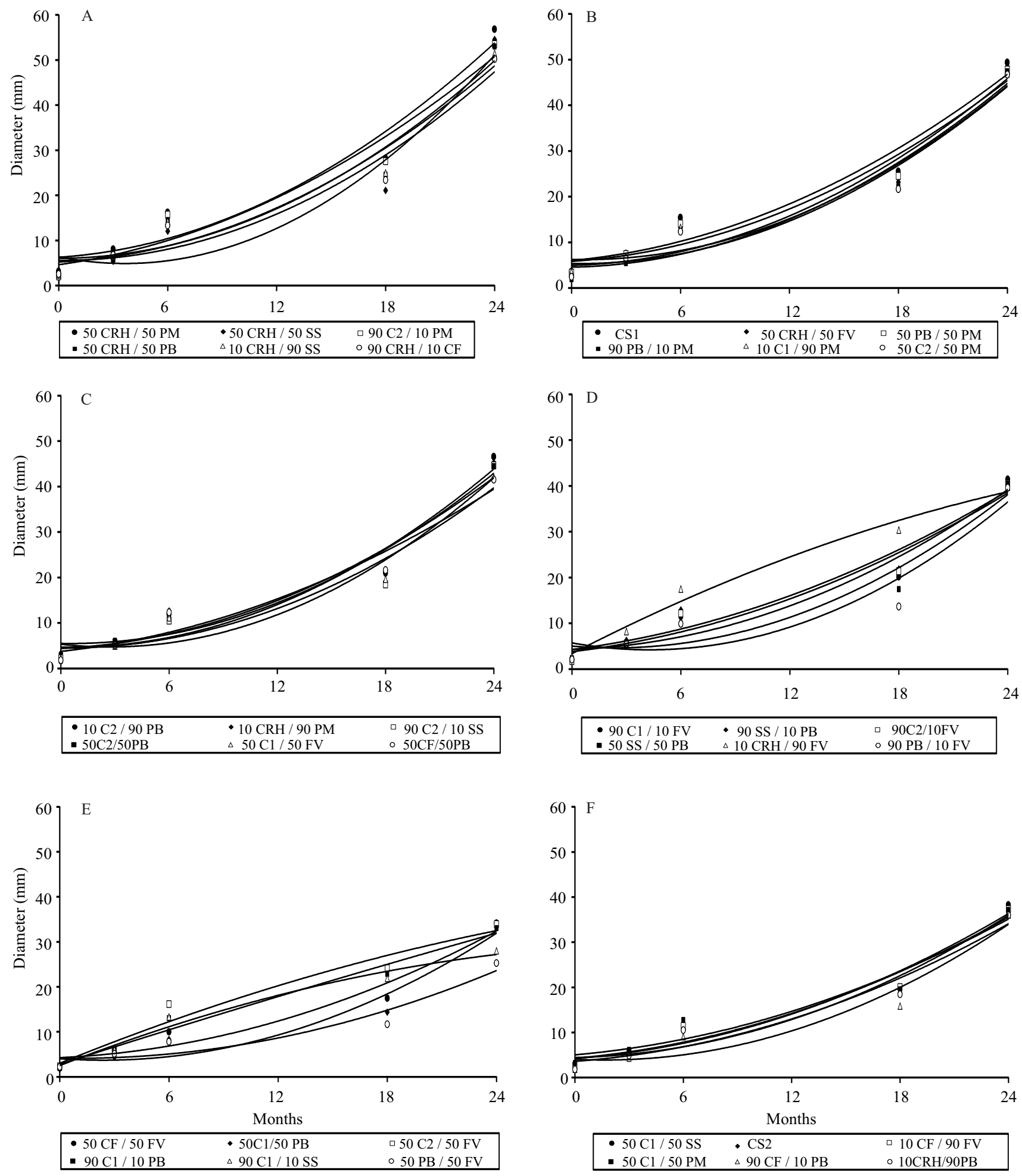

Figure 2. Growth trend for the diameter of Eucalyptus benthamii seedlings produced in 36 substrates and average height at 24 months after planting in the field. CRH, carbonized rice husk; PM, peat moss; SS, sewage sludge; C2, charcoal with granulometry between 3.0-5.0 mm; PB, semi-decomposed pine bark; CF, coconut fiber; CS1, commercial substrate based on peat moss; FV, fine vermiculite; $\mathrm{C}$, charcoal with granulometry between $1.0-3.0 \mathrm{~mm}$; and CS2, commercial substrate based on pine bark. 
Table 5. Increment in diameter (DI), increment in height (HI), transversal area (TA), and basal area (BA) at 24 months after planting in the field of Eucalyptus benthamii seedlings produced in 36 substrates, in the municipality of Pinhais, in the state of Paraná, Brazil ${ }^{(1)}$.

\begin{tabular}{|c|c|c|c|c|}
\hline Substrate $\mathrm{e}^{(2)}$ & $\begin{array}{c}\text { DI } \\
(\mathrm{mm})\end{array}$ & $\begin{array}{l}\mathrm{HIb} \\
(\mathrm{m})\end{array}$ & $\begin{array}{c}\text { Transversal area } \\
\qquad\left(\mathrm{m}^{2}\right)\end{array}$ & $\begin{array}{c}\text { Basal area } \\
\left(\mathrm{m}^{2} \mathrm{ha}^{-1}\right)\end{array}$ \\
\hline $50 \mathrm{CRH} / 50 \mathrm{PM}$ & $53.7 \mathrm{a}$ & $4.9 \mathrm{a}$ & $0.0026 \mathrm{a}$ & $555.9 \mathrm{a}$ \\
\hline $50 \mathrm{CRH} / 50 \mathrm{SS}$ & $51.7 \mathrm{a}$ & $4.5 b$ & $0.0024 \mathrm{a}$ & $401.9 \mathrm{c}$ \\
\hline $90 \mathrm{C} 2$ / 10 PM & $51.5 \mathrm{a}$ & $4.8 \mathrm{a}$ & $0.0023 \mathrm{a}$ & $322.7 d$ \\
\hline $50 \mathrm{CRH} / 50 \mathrm{~PB}$ & $50.2 \mathrm{a}$ & $4.8 \mathrm{a}$ & $0.0023 a$ & $299.6 \mathrm{~d}$ \\
\hline $10 \mathrm{CRH} / 90 \mathrm{SS}$ & $50.1 \mathrm{a}$ & $4.6 \mathrm{~b}$ & $0.0021 \mathrm{a}$ & $453.5 \mathrm{~b}$ \\
\hline $90 \mathrm{CRH} / 10 \mathrm{CF}$ & $47.7 \mathrm{~b}$ & $4.9 \mathrm{a}$ & $0.0020 \mathrm{a}$ & $381.1 \mathrm{c}$ \\
\hline CS1 & $45.8 \mathrm{~b}$ & $4.3 \mathrm{~b}$ & $0.0020 \mathrm{a}$ & $319.7 d$ \\
\hline $50 \mathrm{CRH} / 50 \mathrm{FV}$ & $46.1 \mathrm{~b}$ & $4.2 \mathrm{~b}$ & $0.0019 \mathrm{a}$ & $318.1 d$ \\
\hline $50 \mathrm{~PB} / 50 \mathrm{PM}$ & $44.5 b$ & $4.3 \mathrm{~b}$ & $0.0018 \mathrm{a}$ & $344.6 \mathrm{~d}$ \\
\hline $90 \mathrm{~PB} / 10 \mathrm{PM}$ & $45.5 b$ & $4.5 \mathrm{~b}$ & $0.0018 \mathrm{a}$ & $303.2 d$ \\
\hline $10 \mathrm{C} 1 / 90 \mathrm{PM}$ & $43.3 b$ & $4.0 \mathrm{c}$ & $0.0018 \mathrm{a}$ & $448.0 \mathrm{~b}$ \\
\hline $50 \mathrm{C} 2 / 50 \mathrm{PM}$ & $44.2 \mathrm{~b}$ & $4.6 \mathrm{~b}$ & $0.0018 \mathrm{a}$ & $509.6 \mathrm{a}$ \\
\hline $10 \mathrm{C} 2 / 90 \mathrm{~PB}$ & $44.8 \mathrm{~b}$ & $4.8 \mathrm{a}$ & $0.0018 \mathrm{a}$ & $176.0 \mathrm{f}$ \\
\hline $10 \mathrm{CRH} / 90 \mathrm{PM}$ & $41.9 \mathrm{~b}$ & $4.3 b$ & $0.0017 \mathrm{a}$ & $375.8 \mathrm{c}$ \\
\hline $90 \mathrm{C} 2 / 10 \mathrm{SS}$ & $42.5 b$ & $4.3 \mathrm{~b}$ & $0.0016 \mathrm{a}$ & $356.6 \mathrm{c}$ \\
\hline $50 \mathrm{C} 2 / 50 \mathrm{~PB}$ & $42.9 \mathrm{~b}$ & $4.8 \mathrm{a}$ & $0.0016 \mathrm{a}$ & $223.2 f$ \\
\hline $90 \mathrm{SS} / 10 \mathrm{~PB}$ & $39.8 \mathrm{c}$ & $4.2 \mathrm{~b}$ & $0.0015 b$ & $363.8 \mathrm{c}$ \\
\hline $50 \mathrm{C} 1 / 50 \mathrm{FV}$ & $39.8 \mathrm{c}$ & $3.9 \mathrm{c}$ & $0.0014 b$ & $245.8 \mathrm{e}$ \\
\hline $90 \mathrm{C} 1 / 10 \mathrm{FV}$ & $39.3 \mathrm{c}$ & $4.3 b$ & $0.0014 b$ & $248.6 \mathrm{e}$ \\
\hline $50 \mathrm{CF} / 50 \mathrm{~PB}$ & $39.7 \mathrm{c}$ & $4.5 b$ & $0.0014 b$ & $209.6 f$ \\
\hline $90 \mathrm{C} 2 / 10 \mathrm{FV}$ & $38.7 \mathrm{c}$ & $4.1 \mathrm{~b}$ & $0.0013 \mathrm{~b}$ & $241.9 \mathrm{e}$ \\
\hline $50 \mathrm{SS} / 50 \mathrm{~PB}$ & $38.1 \mathrm{c}$ & $4.3 b$ & $0.0013 b$ & $253.8 \mathrm{e}$ \\
\hline $10 \mathrm{CRH} / 90 \mathrm{FV}$ & $37.0 \mathrm{c}$ & $4.2 \mathrm{~b}$ & $0.0013 b$ & $320.9 \mathrm{~d}$ \\
\hline $90 \mathrm{~PB} / 10 \mathrm{FV}$ & $37.5 \mathrm{c}$ & $4.0 \mathrm{c}$ & $0.0013 b$ & $280.7 \mathrm{e}$ \\
\hline $10 \mathrm{CF} / 90 \mathrm{FV}$ & $39.8 \mathrm{c}$ & $4.3 b$ & $0.0012 b$ & $272.1 \mathrm{e}$ \\
\hline $50 \mathrm{C} 1 / 50 \mathrm{SS}$ & $35.9 \mathrm{c}$ & $4.0 \mathrm{c}$ & $0.0012 b$ & $237.4 \mathrm{e}$ \\
\hline $\mathrm{CS} 2$ & $35.3 \mathrm{c}$ & $4.2 \mathrm{~b}$ & $0.0012 b$ & $213.5 f$ \\
\hline $50 \mathrm{C} 1 / 50 \mathrm{PM}$ & $32.1 \mathrm{~d}$ & $3.7 \mathrm{c}$ & $0.0011 \mathrm{~b}$ & $235.8 \mathrm{e}$ \\
\hline $90 \mathrm{CF} / 10 \mathrm{~PB}$ & $34.2 \mathrm{c}$ & $4.0 \mathrm{c}$ & $0.0011 b$ & $198.7 f$ \\
\hline $10 \mathrm{CRH} / 90 \mathrm{~PB}$ & $34.1 \mathrm{c}$ & $3.8 \mathrm{c}$ & $0.0011 \mathrm{~b}$ & $198.5 \mathrm{f}$ \\
\hline $50 \mathrm{CF} / 50 \mathrm{FV}$ & $31.9 \mathrm{~d}$ & $3.9 \mathrm{c}$ & $0.0009 \mathrm{~b}$ & $187.0 \mathrm{f}$ \\
\hline $50 \mathrm{C} 2 / 50 \mathrm{FV}$ & $31.6 \mathrm{~d}$ & $3.0 \mathrm{~d}$ & $0.0009 \mathrm{~b}$ & $134.8 \mathrm{~g}$ \\
\hline $50 \mathrm{C} 1 / 50 \mathrm{~PB}$ & $31.5 \mathrm{~d}$ & $3.4 \mathrm{~d}$ & $0.0009 b$ & $188.1 \mathrm{f}$ \\
\hline $90 \mathrm{C} 1 / 10 \mathrm{~PB}$ & $31.0 \mathrm{~d}$ & $4.3 \mathrm{~b}$ & $0.0009 \mathrm{~b}$ & $120.5 \mathrm{~g}$ \\
\hline $90 \mathrm{C} 1 / 10 \mathrm{SS}$ & $25.9 \mathrm{e}$ & $3.1 \mathrm{~d}$ & $0.0006 \mathrm{~b}$ & $120.4 \mathrm{~g}$ \\
\hline $50 \mathrm{~PB} / 50 \mathrm{FV}$ & $23.3 \mathrm{e}$ & $3.3 \mathrm{~d}$ & $0.0005 b$ & $71.2 \mathrm{~g}$ \\
\hline
\end{tabular}

${ }^{(1)}$ Means followed by equal letters, in the columns, do not differ by Scott-Knott's test, at $5 \%$ probability. ${ }^{(2)} \mathrm{CRH}$, carbonized rice husk; PM, peat moss; SS, sewage sludge; C2, charcoal with granulometry between 3.0-5.0 mm; PB, semi-decomposed pine bark; CF, coconut fiber; CS1, commercial substrate based on peat moss; FV, fine vermiculite; $\mathrm{C}$, charcoal with granulometry between $1.0-3.0 \mathrm{~mm}$; and CS2, commercial substrate based on pine bark. 
correlated with seedling biometric characteristics in the first measurements, since this relationship weakens as the plants establish themselves and begin their effective growth (Khanal et al., 2018; Shalizi et al., 2019).
Substrates with a greater water holding capacity promote a better growth of $E$. benthamii seedlings during the nursery phase (Kratz et al., 2017). In the field, the morphological characteristics of the seedlings produced in these substrates reflect in a better survival

Table 6. Pearson's correlations between the morphological characteristics ${ }^{(1)}$ of Eucalyptus benthamii seedlings grown in 36 substrates in the nursery and substrate characteristics ${ }^{(2)}$ on seedling survival at $1,2,3,6,12,18$ and 24 months after planting in the field, as well as height, diameter, diameter increment (DI), height increment (HI), individual basal area $\left(\mathrm{BA}_{\mathrm{i}}\right)$, and total basal area $\left(\mathrm{BA}_{\mathrm{T}}\right)$ at 24 months after planting in the field, in the municipality of Pinhais, in the state of Paraná, Brazil.

\begin{tabular}{|c|c|c|c|c|c|c|}
\hline & $\mathrm{AD}$ & $\mathrm{TP}$ & Micro & Macro & $\mathrm{pH}$ & $\mathrm{EC}$ \\
\hline Diameter & $-0.15^{\text {ns }}$ & $0.24^{\mathrm{ns}}$ & $0.39 *$ & $-0.12^{\mathrm{ns}}$ & $-0.52 * *$ & $0.04^{\mathrm{ns}}$ \\
\hline Height & $0.09^{\mathrm{ns}}$ & $0.09^{\mathrm{ns}}$ & $0.33^{*}$ & $-0.22^{\mathrm{ns}}$ & $-0.32^{\mathrm{ns}}$ & $0.24^{\mathrm{ns}}$ \\
\hline $\mathrm{H} / \mathrm{D}$ ratio & $0.45^{* *}$ & $-0.36^{*}$ & $-0.27^{\mathrm{ns}}$ & $-0.11^{\mathrm{ns}}$ & $0.54 * *$ & $0.28^{\mathrm{ns}}$ \\
\hline SDB & $-0.02^{\mathrm{ns}}$ & $0.20^{\mathrm{ns}}$ & $0.41^{*}$ & $-0.18^{\mathrm{ns}}$ & $-0.44 * *$ & $0.16^{\mathrm{ns}}$ \\
\hline $\mathrm{RDB}$ & $-0.03^{\mathrm{ns}}$ & $0.26^{\mathrm{ns}}$ & $0.44 * *$ & $-0.14^{\mathrm{ns}}$ & $-0.49 * *$ & $0.13^{\mathrm{ns}}$ \\
\hline DQI & $-0.11^{\mathrm{ns}}$ & $0.27^{\mathrm{ns}}$ & $0.44 * *$ & $-0.13^{\text {ns }}$ & $-0.53 * *$ & $0.06^{\mathrm{ns}}$ \\
\hline 1 month & $0.00^{\mathrm{ns}}$ & $0.00^{\mathrm{ns}}$ & $0.00^{\mathrm{ns}}$ & $0.00^{\mathrm{ns}}$ & $0.00^{\mathrm{ns}}$ & $0.00^{\mathrm{ns}}$ \\
\hline 2 months & $0.18^{\mathrm{ns}}$ & $0.03^{\mathrm{ns}}$ & $0.26^{\mathrm{ns}}$ & $-0.23^{\mathrm{ns}}$ & $-0.09^{\mathrm{ns}}$ & $0.15^{\mathrm{ns}}$ \\
\hline 3 months & $0.14^{\mathrm{ns}}$ & $0.14^{\mathrm{ns}}$ & $0.28^{\mathrm{ns}}$ & $-0.14^{\mathrm{ns}}$ & $-0.12^{\mathrm{ns}}$ & $0.17^{\mathrm{ns}}$ \\
\hline 6 months & $0.08^{\mathrm{ns}}$ & $0.14^{\mathrm{ns}}$ & $0.47 * *$ & $-0.29^{\mathrm{ns}}$ & $-0.26^{\mathrm{ns}}$ & $0.20^{\mathrm{ns}}$ \\
\hline 12 months & $0.07^{\mathrm{ns}}$ & $0.21^{\mathrm{ns}}$ & $0.47 * *$ & $-0.24^{\mathrm{ns}}$ & $-0.39 *$ & $0.14^{\mathrm{ns}}$ \\
\hline 18 months & $0.07^{\mathrm{ns}}$ & $0.20^{\mathrm{ns}}$ & $0.47 * *$ & $-0.25^{\mathrm{ns}}$ & $-0.41 *$ & $0.14^{\mathrm{ns}}$ \\
\hline 24 months & $0.05^{\mathrm{ns}}$ & $0.23^{\text {ns }}$ & $0.47 * *$ & $-0.21^{\mathrm{ns}}$ & $-0.45^{* *}$ & $0.13^{\text {ns }}$ \\
\hline Diameter & $0.08^{\mathrm{ns}}$ & $0.13^{\text {ns }}$ & $-0.06^{\mathrm{ns}}$ & $0.23^{\text {ns }}$ & $-0.21^{\mathrm{ns}}$ & $0.12^{\mathrm{ns}}$ \\
\hline Height & $0.10^{\mathrm{ns}}$ & $0.18^{\mathrm{ns}}$ & $-0.04^{\mathrm{ns}}$ & $0.25^{\mathrm{ns}}$ & $-0.21^{\mathrm{ns}}$ & $0.11^{\mathrm{ns}}$ \\
\hline DI & $0.11^{\mathrm{ns}}$ & $0.16^{\mathrm{ns}}$ & $0.01^{\mathrm{ns}}$ & $0.18^{\mathrm{ns}}$ & $-0.17^{\mathrm{ns}}$ & $0.13^{\text {ns }}$ \\
\hline $\mathrm{HI}$ & $0.06^{\mathrm{ns}}$ & $0.18^{\mathrm{ns}}$ & $-0.03^{\mathrm{ns}}$ & $0.23^{\mathrm{ns}}$ & $-0.18^{\mathrm{ns}}$ & $0.04^{\mathrm{ns}}$ \\
\hline $\mathrm{BA}_{\mathrm{i}}$ & $0.11^{\mathrm{ns}}$ & $0.19^{\mathrm{ns}}$ & $0.02^{\mathrm{ns}}$ & $0.21^{\mathrm{ns}}$ & $-0.23^{\mathrm{ns}}$ & $0.13^{\mathrm{ns}}$ \\
\hline \multirow[t]{2}{*}{$\mathrm{BA}_{\mathrm{T}}$} & $0.13^{\mathrm{ns}}$ & $0.13^{\text {ns }}$ & $0.23^{\mathrm{ns}}$ & $-0.06^{\mathrm{ns}}$ & $-0.31^{\mathrm{ns}}$ & $0.24^{\mathrm{ns}}$ \\
\hline & Diameter & Height & $\mathrm{H} / \mathrm{D}$ ratio & SDB & RDB & DQI \\
\hline 1 month & $0.00^{\mathrm{ns}}$ & $0.00^{\mathrm{ns}}$ & $0.00^{\mathrm{ns}}$ & $0.00^{\mathrm{ns}}$ & $0.00^{\mathrm{ns}}$ & $0.00^{\mathrm{ns}}$ \\
\hline 2 months & $0.26^{\mathrm{ns}}$ & $0.35^{*}$ & $0.06^{\mathrm{ns}}$ & $0.26^{\mathrm{ns}}$ & $0.31^{\mathrm{ns}}$ & $0.25^{\text {ns }}$ \\
\hline 3 months & $0.32^{\mathrm{ns}}$ & $0.38^{*}$ & $-0.04^{\mathrm{ns}}$ & $0.32^{\text {ns }}$ & $0.37 *$ & $0.32^{\text {ns }}$ \\
\hline 6 months & $0.54 * *$ & $0.52 * *$ & $-0.27^{\mathrm{ns}}$ & $0.49 * *$ & $0.53 * *$ & $0.52 * *$ \\
\hline 12 months & $0.58 * *$ & $0.53 * *$ & $-0.32^{\text {ns }}$ & $0.54 * *$ & $0.58 * *$ & $0.57 * *$ \\
\hline 18 months & $0.56^{* *}$ & $0.52 * *$ & $-0.33^{\mathrm{ns}}$ & $0.52 * *$ & $0.55^{* *}$ & $0.55^{*}$ \\
\hline 24 months & $0.57 * *$ & $0.50 * *$ & $-0.38 *$ & $0.52 * *$ & $0.56^{* *}$ & $0.56^{* *}$ \\
\hline Diameter & $0.34 *$ & $0.36^{*}$ & $-0.12^{\mathrm{ns}}$ & $0.38^{*}$ & $0.37 *$ & $0.36^{*}$ \\
\hline Height & $0.24^{\mathrm{ns}}$ & $0.22^{\mathrm{ns}}$ & $-0.15^{\text {ns }}$ & $0.27^{\mathrm{ns}}$ & $0.27^{\mathrm{ns}}$ & $0.27^{\mathrm{ns}}$ \\
\hline DI & $0.30^{\text {ns }}$ & $0.31^{\mathrm{ns}}$ & $-0.12^{\mathrm{ns}}$ & $0.33^{\text {ns }}$ & $0.33^{\text {ns }}$ & $0.31^{\mathrm{ns}}$ \\
\hline HI & $0.10^{\mathrm{ns}}$ & $0.05^{\mathrm{ns}}$ & $-0.11^{\mathrm{ns}}$ & $0.11^{\mathrm{ns}}$ & $0.11^{\mathrm{ns}}$ & $0.11^{\mathrm{ns}}$ \\
\hline $\mathrm{BA}_{\mathrm{i}}$ & $0.38^{*}$ & $0.37^{*}$ & $-0.17^{\mathrm{ns}}$ & $0.39^{*}$ & $0.39^{*}$ & $0.39 *$ \\
\hline $\mathrm{BA}_{\mathrm{T}}$ & $0.53 * *$ & $0.55^{* *}$ & $-0.24^{\mathrm{ns}}$ & $0.55^{* *}$ & $0.55 * *$ & $0.54 * *$ \\
\hline
\end{tabular}

${ }^{(1)}$ Morphological characteristics: diameter; height; H/D ratio, height/diameter ratio; SDB, shoot dry biomass; RDB, root dry biomass; and DQI, Dickson quality index. ${ }^{(2)}$ Substrate characteristics: AD, apparent density; TP, total porosity; Micro, microporosity; Macro, macroporosity; pH; and EC, electrical conductivity. 
and initial growth. Diameter, as well as biomass, are important morphological parameters to indicate seedling quality, and may be key to establish new parameters to determine the quality of E. benthamii seedlings.

\section{Conclusions}

1. Substrates affect the morphological characteristics of Eucalyptus benthamii seedlings in the nursery phase, and these characteristics are important for plant survival and initial growth after planting in the field.

2. Seedlings that show $100 \%$ survival after 24 months under field conditions were grown in substrates with a higher proportion of fine vermiculite, sewage sludge, and peat moss in the nursery phase, that is, in substrates with a higher microporosity.

3. The lowest survival of $60 \%$ is observed for seedlings produced in substrates with the predominance of charcoal with a granulometry between 1.0-3.0 or $3.0-5.0 \mathrm{~mm}$ and pine bark.

4. The initial growth of seedlings is not influenced by substrate characteristics, but diameter after 24 months is directly affected by seedling morphological characteristics at the time of field planting.

5. The significant correlation between the survival of seedlings at 6 months and their diameter at the end of nursery period evidences the importance of this characteristic for the establishment of the plant in the field, regardless of the used substrate.

\section{Acknowledgments}

To Embrapa Florestas and Fazenda Canguiri of Universidade Federal do Paraná (UFPR), for support; and to Centro de Assessoria de Publicação Acadêmica (Capa) of UFPR, for assistance with the translation into English.

\section{References}

ALVARES, C.A.; STAPE, J.L.; SENTELHAS, P.C.; GONÇALVES, J.L. de M.; SPAROVEK, G. Köppen's climate classification map for Brazil. Meteorologische Zeitschrift, v.22, p.711-728, 2013. DOI: https://doi.org/10.1127/09412948/2013/0507.

ARNOLD, R.; LI, B.; LUO, J.; BAI, F.; BAKER, T. Selection of cold-tolerant Eucalyptus species and provenances for inland frost-susceptible, humid subtropical regions of southern China.
Australian Forestry, v.78, p.180-193, 2015. DOI: https://doi.org/1 0.1080/00049158.2015.1063471.

BRASIL. Ministério da Agricultura, Pecuária e Abastecimento. Instrução Normativa SDA no 17, de 21 de maio 2007. 2007. Available at: <http://www.agricultura.gov.br/assuntos/insumosagropecuarios/insumos-agricolas/fertilizantes/legislacao/in-17de-21-05-2007-aprova-metodo-substrato.pdf $>$. Accessed on: May 282020 .

CAVALETT, O.; SLETTMO, S.N.; CHERUBINI, F. Energy and environmental aspects of using eucalyptus from Brazil for energy and transportation services in Europe. Sustainability, v.10, art.4068, 2018. DOI: https://doi.org/10.3390/su10114068.

DIAS JÚNIOR, A.F.; COSTA JÚNIOR, D.S. da; ANDRADE, A.M. de; OLIVEIRA, E. de; LANA, A.Q.; BRITO, J.O. Quality of Eucalyptus wood grown in Rio de Janeiro state for bioenergy. Floresta e Ambiente, v.23, p.435-442, 2016. DOI: https://doi.org/10.1590/2179-8087.140315.

ELLI, E.F.; SENTELHAS, P.C.; FREITAS, C.H. de; CARNEIRO, R.L.; ALVARES, C.A. Assessing the growth gaps of Eucalyptus plantations in Brazil - magnitudes, causes and possible mitigation strategies. Forest Ecology and Management, v.451, art.117464, 2019. DOI: https://doi.org/10.1016/j.foreco.2019.117464.

EUFRADE JUNIOR, H.J.; MELO, R.X. de; SARTORI, M.M.P.; GUERRA, S.P.S.; BALLARIN, A.W. Sustainable use of eucalypt biomass grown on short rotation coppice for bioenergy. Biomass and Bioenergy, v.90, p.15-21, 2016. DOI: https://doi.org/10.1016/j. biombioe.2016.03.037.

GABIRA, M.M.; SILVA, R.B.G. da; MATEUS, C. de M.D'A.; VILLAS BOAS, R.L.; SILVA, M.R. da. Effects of water management and composted sewage sludge substrates on the growth and quality of clonal eucalyptus seedlings. Floresta, v.50, p.1307-1314, 2020. DOI: https://doi.org/10.5380/rf.v50i2.62952.

GONZAGA, M.I.S.; MACKOWIAK, C.; ALMEIDA, A.Q. de; CARVALHO JÚNIOR, J.I.T. de. Sewage sludge derived biochar and its effect on the growth and morphological traits of Eucalyptus grandis W.Hill ex Maiden seedlings. Ciência Florestal, v.28, p.687-695, 2018. DOI: https://doi.org/10.5902/1980509832067.

GROSSNICKLE, S.C.; EL-KASSABY, Y.A. Bareroot versus container stocktypes: a performance comparison. New Forests, v.47, p.1-51, 2016. DOI: https://doi.org/10.1007/s11056-015-94766.

IUSS WORKING GROUP WRB. World reference base for soil resources 2014: international soil classification system for naming soils and creating legends for soil maps: update 2015. Rome: FAO, 2015. 182p. (FAO. World Soil Resources Report 106).

KHANAL, P.N.; DEAN, T.J.; ROBERTS, S.D.; GREBNER, D.L.; STRAKA, T.J. Explaining first-year seedling survival from quality distributions of bare-root seedlings and microsites in industrial plantations. Open Journal of Forestry, v.8, p.362-379, 2018. DOI: https://doi.org/10.4236/ojf.2018.83023.

KRATZ, D.; NOGUEIRA, A.C.; WENDLING, I.; MELLEK, J.E. Physic-chemical properties and substrate formulation for Eucalyptus seedlings production. Scientia Forestalis, v.45, p.6376, 2017. DOI: https://doi.org/10.18671/scifor.v45n113.06. 
KRATZ, D.; WENDLING, I. Crescimento de mudas de Eucalyptus camaldulensis em substratos à base de casca de arroz carbonizada. Revista Ceres, v.63, p.348-354, 2016. DOI: https://doi.org/10.1590/0034-737X201663030011.

KRATZ, D.; WENDLING, I.; NOGUEIRA, A.C.; SOUZA, P.V.D. de. Substratos renováveis na produção de mudas de Eucalyptus benthamii. Ciência Florestal, v.23, p.607-621, 2013. DOI: https://doi.org/10.5902/1980509812345.

KRATZ, D.; WENDLING, I.; PIRES, P.P. Miniestaquia de Eucalyptus benthamii x E. dunnii em substratos a base de casca de arroz carbonizada. Scientia Forestalis, v.40, p.547-556, 2012.

MANCA, A.; SILVA, M.R. da; GUERRINI, I.A.; FERNANDES, D.M.; VILLAS BÔAS, R.L.; SILVA, L.C. da; FONSECA, A.C. da; RUGGIU, M.C.; CRUZ, C.V.; SIVISACA, D.C.L.; MATEUS, C. de M.D'.A.; MURGIA, I.; GRILLI, E.; GANGA, A.; CAPRA, G.F. Composted sewage sludge with sugarcane bagasse as a commercial substrate for Eucalyptus urograndis seedling production. Journal of Cleaner Production, v.269, art.122145, 2020. DOI: https://doi.org/10.1016/j.jclepro.2020.122145.

MENUCELLI, J.R.; AMORIM, E.P.; FREITAS, M.L.M.; ZANATA, M.; CAMBUIM, J.; MORAES, M.L.T. de; YAMAJI, F.M.; SILVA JÚNIOR, F.G. da; LONGUI, E.L. Potential of Hevea brasiliensis clones, Eucalyptus pellita and Eucalyptus tereticornis wood as raw materials for bioenergy based on higher heating value. Bioenergy Research, v.12, p.992-999, 2019. DOI: https://doi.org/10.1007/s12155-019-10041-6.

MIETH, P.; ARAUJO, M.M.; FERMINO, M.H.; AIMI, S.C.; GOMES, D.R.; VILELLA, J. de M. Ground peach pits: alternative substrate component for seedling production. Journal of Forestry Research, v.30, p.1779-1791, 2019. DOI: https://doi.org/10.1007/ s11676-018-0740-4.

PERRI, D.; GOROSITO, N.; FERNANDEZ, P.; BUTELER, M. Plant-based compounds with potential as push-pull stimuli to manage behavior of leaf-cutting ants. Entomologia Experimentalis et Applicata, v.163, p.150-159, 2017. DOI: https://doi.org/10.1111/eea.12574.
RESQUIN, F.; NAVARRO-CERRILLO, R.M.; RACHIDCASNATI, C.; HIRIGOYEN, A.; CARRASCO-LETELIER, L.; DUQUE-LAZO, J. Allometry, growth and survival of three eucalyptus species (Eucalyptus benthamii Maiden and Cambage, E. dunnii Maiden and E. grandis Hill ex Maiden) in highdensity plantations in Uruguay. Forests, v.9, art.745, 2018. DOI: https://doi.org/10.3390/f9120745.

RIIKONEN, J.; LUORANEN, J. Seedling production and the field performance of seedlings. Forests, v.9, art.740, 2018. DOI: https://doi.org/10.3390/f9120740.

SANTOS, H.G. dos; JACOMINE, P.K.T.; ANJOS, L.H.C. dos; OLIVEIRA, V.Á. de; LUMBRERAS, J.F.; COELHO, M.R.; ALMEIDA, J.A. de; CUNHA, T.J.F.; OLIVEIRA, J.B. de. Sistema brasileiro de classificação de solos. 3.ed. rev. e ampl. Brasília: Embrapa, 2013. 353p.

SHALIZI, M.N.; GOLDFARB, B.; BURNEY, O.T.; SHEAR, T.H. Effects of five growing media and two fertilizer levels on polybagraised Camden whitegum (Eucalyptus benthamii Maiden \& Cambage) seedling morphology and drought hardiness. Forests, v.10, art.543, 2019. DOI: https://doi.org/10.3390/f10070543.

STUEPP, C.A.; WENDLING, I.; KOEHLER, H.S.; ZUFFELLATO-RIBAS, K.C. Quality of clonal plants of Piptocarpha angustifolia in different renewable substrates and seasons of the year. Pesquisa Agropecuária Brasileira, v.51, p.1821-1829, 2016. DOI: https://doi.org/10.1590/S0100$204 X 2016001100004$.

WANG, L.; HU, H.; TENG, W.; HOU, W.; WEI, J.; GOODALE, U.M.; BAI, T.; ZHANG, B. Orthogonal fertilization tests designed to optimize the quality of Eucalyptus seedlings. Journal of Plant Nutrition, v.41, p.1507-1521, 2018. DOI: https://doi.org/10.1080/0 1904167.2018.1458869.

YASIN, M.; JABRAN, K.; AFZAL, I.; IQBAL, S.; NAWAZ, M.A.; MAHMOOD, A.; ASIF, M.; NADEEM, M.A.; RAHMAN, Z.U.; ADNAN, M.; SIDDIQUI, M.; SHAHID, M.Q.; ANDREASEN, C. Industrial sawdust waste: an alternative to soilless substrate for garlic (Allium sativum L.). Journal of Applied Research on Medicinal and Aromatic Plants, v.18, art.100252, 2020. DOI: https://doi.org/10.1016/j.jarmap.2020.100252. 